\title{
Perceived control moderates the relationship between anxiety and in-hospital complications after ST segment elevation myocardial infarction
}

This article was published in the following Dove Press journal: Journal of Multidisciplinary Healthcare

\author{
Mohannad Eid AbuRuz \\ Clinical Nursing Department, College \\ of Nursing, Applied Science Private \\ University, Amman, Jordan
}

\begin{abstract}
Purpose: Anxiety is a common psychological response after acute myocardial infarction and might be associated with higher levels of in-hospital complications. Perceived control might moderate this relationship, but the effect of this method has not been checked in developing countries. Therefore, the objectives of this study were as follows: 1) to check if anxiety was an independent predictor of in-hospital complications after acute myocardial infarction; and 2) to check if perceived control moderates the relationship between anxiety and in-hospital complications after acute myocardial infarction.
\end{abstract}

Patients and methods: This was a prospective observational study among 500 patients with a confirmed diagnosis of ST segment elevation myocardial infarction recruited from three private hospitals in Amman, Jordan. Anxiety was measured by the Anxiety subscale of Hospital Anxiety and Depression Scale, and perceived control was measured by the Arabic version of the Control Attitude Scale-Revised.

Results: One hundred and forty patients (28\%) developed at least one complication during hospitalization. Two hundred and fifty-five patients had low anxiety scores $(\leq 7)$, and 245 patients had high anxiety scores $(\geq 8)$. Patients with high levels of perceived control had lower levels of anxiety (mean [SD]; 5.3 [3.6] vs 14.1 [6.3], P<0.001) than those with low perceived control. In logistic regression, anxiety was an independent predictor of in-hospital complications after controlling for sociodemographic and clinical variables (odds ratio: 1.24, 95\% CI, 1.08-1.41, $P<0.01)$. Moreover, the interaction of anxiety and perceived control was a significant predictor of complications.

Conclusion: Anxiety was associated with increased risk of in-hospital complications after acute myocardial infarction independent of sociodemographic and clinical variables. Perceived control had a moderating effect for this association since the combination of low perceived control and high anxiety scores was associated with the greatest risk for complications. Supporting patients' levels of perceived control can decrease complications, morbidities, and mortality after acute myocardial infarction.

Keywords: anxiety, perceived control, acute myocardial infarction, complications

\section{Introduction}

In 2013, cardiovascular disease (CVD) caused >17 million deaths globally, remaining the number one cause of death worldwide. ${ }^{1}$ More than 92 million Americans have at least one type of CVD. ${ }^{1}$ It is estimated that by $2030,44 \%$ of the US population will have some form of CVD. ${ }^{1}$ Developing (low and middle income) countries account for the highest percentage of deaths $(80 \%)$ due to CVD globally; in Jordan, it is the leading cause of death, resulting in $35 \%$ of all mortalities. ${ }^{2}$ 
Coronary heart disease (CHD) is one of the most common CVDs and makes up more than half of all CVD events. ${ }^{1}$ Approximate every 25 seconds an American will have a CVD event. ${ }^{3}$ Acute myocardial infarction (AMI) is the primary outcome of CHD. ${ }^{2} \mathrm{AMI}$ is a leading cause of death in Jordan. ${ }^{3}$ According to the World Health Organization, CHD deaths account for one-fifth of all deaths in Jordan (131 per $100,000){ }^{4}$

Psychological responses including anxiety are common after AMI. The incidence rate of anxiety symptoms after AMI is high and might reach up to $70 \%-80 \%$, and more than a quarter have higher anxiety levels than psychiatric patients. ${ }^{2,5}$ Anxiety was considered a serious risk factor equivalent to more traditional risk factors such as smoking and hypertension. ${ }^{3}$ In addition, new studies showed that anxiety after AMI is considered a global health problem, which usually affects females more than males. ${ }^{2}$

While anxiety is an intrinsically negative health condition in itself, it can have beneficial health impacts when it induces patients to seek treatment. However, it may exert severe negative outcomes including increased morbidity and mortality due to in-hospital ischemic and arrhythmic complications (ie, ventricular tachycardia and ventricular fibrillation), which frequently occur in the first 72 hours after the event. ${ }^{2,6}$

Most studies on the effect of anxiety on in-hospital complications showed that anxiety increased the complications or was an independent predictor of these complications after controlling for sociodemographic and clinical variables, ${ }^{2,6-15}$ while other studies ${ }^{5,16-18}$ showed that anxiety did not have any relationship with complications. Only one study ${ }^{19}$ showed that anxiety was associated with a survival advantage. The most recent study ${ }^{2}$ showed that anxiety increased the occurrence of the complications by $20 \%$, independent of sociodemographic and clinical variables. Therefore, application of different methods to control the effect of anxiety on in-hospital complications after AMI is warranted.

One of these methods, which is relatively new in the investigations, is the perceived control (PC) of cardiac illness. PC is defined as "an individual's belief that he or she has the resources required to cope with negative events in a way that positively influences their adversive nature." ${ }^{20} \mathrm{PC}$ can be improved by different methods, including education and counseling. ${ }^{20}$

After an AMI event, patients' adaptation, psychosocial recovery, and quality of life depend more on psychological rather than physiological factors. ${ }^{20-22} \mathrm{PC}$ has been negatively correlated with anxiety in various cardiac populations, including AMI, cardiac surgery, and heart failure. PC has been found to moderate the relationship between anxiety and complications after AMI, ${ }^{10}$ and it was an independent predictor of anxiety in AMI, heart failure, and CHD patients. ${ }^{20}$

To my knowledge, all studies that checked the effect of PC in cardiac populations were conducted in developed countries. Therefore, the purpose of this study was to check the hypothesis that PC moderates the relationship between anxiety and in-hospital complications after AMI in a developing country.

\section{Patients and methods}

\section{Research design, sample, and setting}

A prospective comparative design was used to test the hypotheses of this study. Patients were recruited from three private hospitals in Amman, Jordan. Patients were enrolled in the study subject to the following inclusion criteria: 1) confirmed diagnosis of ST segment elevation MI (STEMI) by ECG changes and elevated cardiac enzymes by a cardiologist; 2) older than 18 years; 3 ) pain-free and hemodynamically stable during the interview; 4) not lacking decision-making capacity to sign informed consent or to answer the questionnaire; and 5) free of noncardiac life-threatening comorbidities as stroke, shock, and sepsis. Patients who developed in-hospital complications before anxiety was measured during the interview were excluded from the study to ensure the temporality aspect and to avoid increased anxiety due to the longitudinal development of these complications.

A priori sample size calculator ${ }^{23}$ was used to calculate the required sample size. The assumptions were a medium effect size of 0.15 , a power of 0.95 , an $\alpha$ of 0.01 , and 15 independent variables for the regression analysis. Accordingly, the needed number to reach statistical significance based on these assumptions was 252 participants. Therefore, 500 participants were considered sufficient for the planned analyses (Figure 1).

\section{Ethical considerations}

The study was approved by the institutional review board (IRB) at the Applied Science Private University, Amman, Jordan. Formal letters were sent to the selected hospitals by the University President along with the IRB approval asking them to facilitate the study fieldwork. After that, the principal investigator met with the medical directors, who conveyed their acceptance of the IRB approval letter and gave permission to conduct the study. 


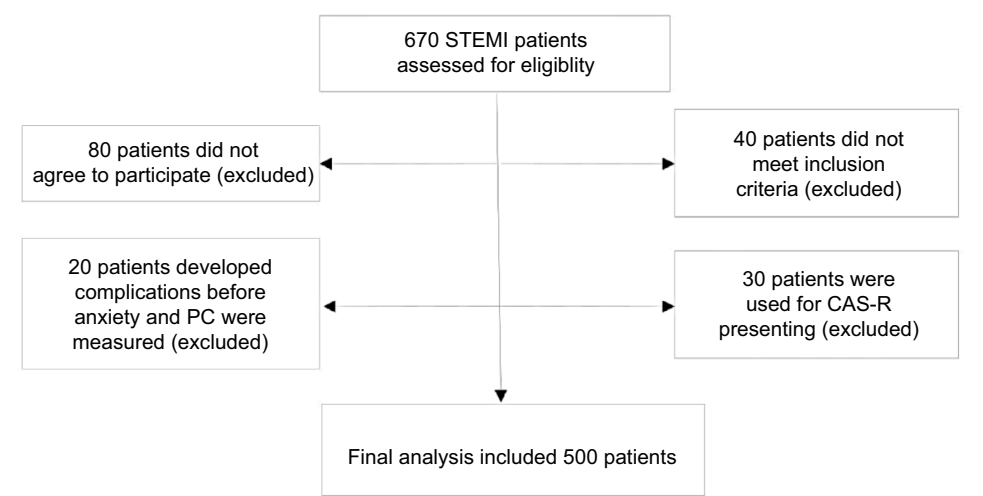

Figure I Patient flow diagram.

Note: A total of 500 patients were included in the final analyses.

Abbreviations: CAS-R, Control Attitude Scale-revised; PC, perceived control; STEMI, ST segment elevation myocardial infarction.

\section{Procedure}

Three trained cardiovascular research assistant conducted the data collection. They approached every patient with a confirmed diagnosis of STEMI in the selected hospitals and explained the study in detail, assuring the patients that their participation was voluntary and they could withdraw at any time. Patients who agreed to participate signed an informed consent form. During the first 72 hours of the admission (mean \pm SD; $37 \pm 15$ ), when the patients were hemodynamically stable, they were interviewed. Their sociodemographic data was recorded, and then the Anxiety subscale of the Hospital Anxiety and Depression Scale (HADS) and the translated (Arabic) Control Attitude Scale-Revised (CAS-R) were administered. In-hospital complications, all other comorbidities, and necessary data were extracted from medical recorded after discharge.

\section{Measurement of variables}

\section{Sociodemographic and clinical characteristics}

Medical records were reviewed by research assistants after discharge to collect the following information: age, gender, medications used in emergency department and in ICU, history of hypertension, diabetes mellitus, previous AMI, smoking, left ventricular ejection fraction, admission vital signs, severity of chest pain, and body mass index.

\section{Anxiety}

Anxiety was measured by the Arabic version of HADS. Previous studies ${ }^{24-28}$ demonstrated the psychometric proprieties of this version. The Cronbach's $\alpha$ was 0.78 . Anxiety subscale has seven items that were rated by patients on a
0-3 scale, with 3 indicating higher symptom frequency and severity. The total score ranged from $0-21$. Anxiety scores between 0 and 7 were considered normal, between 8 and 10 were considered mild, between 11 and 14 were considered moderate, and between 15 and 21 were considered severe. $^{24-28}$

\section{In-hospital complications}

In-hospital complications were defined as in previous studies $^{2,3,5,6,10,13,15}$ as the occurrence of any of the following during hospitalization: 1) reinfarction; 2) acute recurrent ischemia; 3) sustained ventricular tachycardia or any ventricular tachycardia requiring intervention; 4) ventricular fibrillation; (5) acute pulmonary edema; 6) cardiogenic shock; and 7) in-hospital death.

\section{PC}

There is no validated tool in Arabic language that measures PC. For this reason, the principal investigator used the standard World Health Organization method (translation and backtranslation to ensure fidelity) ${ }^{29}$ to translate the CAS-R and used it in this study. This method consisted of the flowing steps: 1) forward translation; 2) expert backtranslation; 3) pretesting and cognitive interviewing; and 4) final version.

The original (ie, English-language) CAS-R consists of eight Likert-type items answered by the patients within two minutes in previous studies. ${ }^{5,20}$ Possible answers range from 1 "totally disagree" to 5 "totally agree". Total score ranged from 8-40, with higher scores indicating higher levels of PC..$^{5,20}$ The validity and reliability of this instrument have 
been empirically demonstrated. ${ }^{20}$ The Cronbach's alpha coefficients for CHD, AMI, and heart failure patients were $0.73,0.72$, and 0.76 , respectively. Construct validity was supported by factor analysis and hypothesis testing. Hypothesis testing in each of the three cardiac samples (CHD, AMI, and heart failure) showed that higher levels of PC decreased anxiety. Another study showed an acceptable level of internal consistency with a Cronbach's $\alpha$ of 0.68 in a smaller sample of 171 patients with acute coronary syndrome. ${ }^{5}$

Prior to data collection, the CAS-R was translated to Arabic language by an expert professor in critical care, then an expert panel of three professors (one in critical care, one in health, and one in instrument development and translation) conducted backtranslation. The purpose of this step was to identify and resolve any inadequate expressions or concepts in the translation. No significant adjustments were required. Next, pretesting and cognitive interviewing were done on 30 AMI patients, who were excluded from the sample. Respondents were asked about any word they did not understand as well as any words or expression that they found unacceptable. These questions reflected the clarity of the instrument. Moreover, this step was done to determine the duration needed to complete the questionnaire. No comments/questions were raised by the participants, and the required time to complete the instrument was between 2-3 minutes.

The new translated instrument (the final version) was used to collect information regarding PC. Cronbach's $\alpha$ for the Arabic version was 0.75 . The construct validity also was checked by hypothesis testing. Patients with high PC have lower levels of anxiety compared to those with low PC. There are no published mean norms for this tool; therefore, researchers usually use the median of their samples as a cutoff point. ${ }^{5,20}$ Consequently, patients were classified as having high and low PC based on the median of the sample in this study, which was 31 .

\section{Data analysis}

Data were analyzed using SPSS software version 21 (IBM Corp., Armonk, NY, USA). Any $P$-value $<0.05$ was considered statistically significant. Sociodemographic and clinical characteristics were described by mean \pm SD or frequencies and percentages, based on the level of anxiety. To decrease the likelihood that the association between anxiety and complications could be explained by differences in treatments at baseline or sociodemographic and clinical characteristics between low (0-7) and high (8-21) anxiety groups, patients' data were examined either using Student's $t$-test for continuous variables and $X^{2}$ for categorical variables for any differences. All variables that were different between the groups were controlled for in subsequent analyses.

Multiple logistic regression was used to test the hypothesis that PC moderates the association between anxiety and in-hospital complications. The results are presented as odds ratios and $95 \% \mathrm{CIs}$ in relation to demographics (age and gender), clinical characteristics (history of diabetes, history of hypertension, history of previous AMI, history of smoking, use of beta blocker, aspirin, or anxiolytics in emergency department, and left ventricular ejection fraction), and psychological status (anxiety scores, PC scores, and anxiety * PC interaction). After the demographic and clinical variables were entered in the model, anxiety and PC scores were entered first, followed by the interaction form, to determine how much anxiety and PC improved the predictive model. There was no multicollinearity between the variables, since the variance inflation factor was $<3 .^{30}$

\section{Results}

\section{Sociodemographic data}

A total of 500 patients participated in the study, comprising 338 men and 162 women. A total of 140 patients $(28 \%)$ developed at least one complication during hospitalization (Table 1). Patients with higher levels of anxiety were more likely to develop complications $\left(X^{2}=23.74, P<0.001\right)$ and were more likely to be smokers $\left(X^{2}=6.86, P<0.05\right)$ than those with lower levels of anxiety. All other variables did not differ between the high- and low-anxiety groups. Patients with high levels of PC had lower levels of anxiety than those with low PC (mean [SD], 5.3 [3.6] vs 14.1 [6.3], $P<0.001$ ). Sociodemographics and other comorbidities relative to anxiety levels are presented in Table 2. Only two clinical variables differed between high-anxiety and low-anxiety groups. Patients in the high-anxiety group received beta blockers and antianxiety medication more often than those in the low-anxiety group (Table 3).

Table I Specific complications developed and their percentages

\begin{tabular}{ll}
\hline Complications developed & Number of patients (\%) \\
\hline Acute recurrent ischemia & $63(45)$ \\
Sustained ventricular tachycardia & $15(10.7)$ \\
Ventricular fibrillation & $12(8.6)$ \\
Reinfarction & $11(7.6)$ \\
Cardiogenic shock & $7(5.0)$ \\
In-hospital death & $6(4.3)$ \\
Pulmonary edema & $5(3.6)$ \\
\hline
\end{tabular}

Note: aMore than one patient developed more than one complication. 
Table 2 Sociodemographic characteristics of the sample $(\mathrm{N}=500)$

\begin{tabular}{lll}
\hline Characteristics & $\begin{array}{l}\text { High anxiety } \\
(\mathbf{n = 2 4 5 )}\end{array}$ & $\begin{array}{l}\text { Low anxiety } \\
(\mathbf{n}=\mathbf{2 5 5})\end{array}$ \\
\hline $\begin{array}{l}\text { Age, years } \\
\text { Gender }\end{array}$ & $66.5 \pm 10.58$ & $68.2 \pm 1 \mathrm{I} .15$ \\
$\quad$ Male & $171(69.8)$ & $167(65.5)$ \\
$\quad$ Female & $74(30.2)$ & $88(34.5)$ \\
History of DM & $98(40.0)$ & $99(38.8)$ \\
History of HTN & $179(73.1)$ & $192(75.3)$ \\
History of previous AMI & $163(66.5)$ & $160(62.7)$ \\
History of smoking & $184(75.1)$ & $163(63.9)^{*}$ \\
Severity of chest pain & $5.26 \pm 2.49$ & $5.28 \pm 2.03$ \\
Ejection fraction & $48.57 \pm 8.39$ & $48.17 \pm 8.7$ \\
Development of complications & $92(36.7)$ & $48(18.8)^{* * *}$ \\
\hline
\end{tabular}

Notes: Values are presented as mean \pm SD or $\mathrm{n}(\%)$, *significant at $P<0.05$, ****significant at $P<0.001$.

Abbreviations: DM, diabetes mellitus; HTN, hypertension; AMI, acute myocardial infarction.

Table 3 Treatment received during hospitalization $(n=500)$

\begin{tabular}{lll}
\hline Treatments & $\begin{array}{l}\text { High anxiety } \\
(\mathbf{n = 2 4 5 )}\end{array}$ & $\begin{array}{l}\text { Low anxiety } \\
(\mathbf{2 5 5})\end{array}$ \\
\hline Thrombolytic agents & $101(41.2)$ & $89(34.9)$ \\
Beta blocker & $125(51.0)$ & $107(41.9)^{*}$ \\
Aspirin & $221(90.2)$ & $216(84.7)$ \\
Antianxiety & $140(57.1)$ & $110(43.1)^{*}$ \\
Coronary artery bypass graft & $22(8.9)$ & $35(13.7)$ \\
Angioplasty & $152(62.0)$ & $164(64.3)$ \\
\hline
\end{tabular}

Notes: Values are presented as $n(\%)$. *Significant at $P<0.05$.

\section{Hypothesis testing}

Among all variables entered in the logistic regression (age, gender, left ventricular ejection fraction, history of diabetes, history of hypertension, history of previous AMI, history of smoking, use of beta blocker, aspirin, or anxiolytics in emergency department, anxiety scores, PC scores, and anxiety control interaction), only history of smoking, history of previous AMI, anxiety scores, PC scores, left ventricular ejection fraction, and the interaction of anxiety and $\mathrm{PC}$ were significant predictors of complications (Table 4). PC moderated the association between anxiety and complications since the interaction between anxiety and PC was a significant predictor of complications. Patients with high anxiety and low PC had the highest occurrence of complications $(40 \%$ vs $11.4 \%, P<0.001$ ), while this was not significant for the low-anxiety group (Figure 2 ).

\section{Discussion}

The results of this study, which was conducted in a developing country, showed that PC moderated the association between anxiety and complications after STEMI. Moreover, this study showed that anxiety increased the risk of complications
Table 4 Logistic regression analysis for predictors of in-hospital complications

\begin{tabular}{lllll}
\hline Predictors & $\begin{array}{c}\text { Odds } \\
\text { ratio }\end{array}$ & Wald & 95\% Cl & P-value \\
\hline History of smoking & 1.12 & 10.16 & $1.06-1.19$ & 0.008 \\
History of previous AMI & 2.18 & 8.43 & $1.21-3.81$ & 0.005 \\
Anxiety scores & 1.24 & 9.70 & $1.08-1.41$ & 0.006 \\
Perceived control & 0.91 & 7.21 & $0.85-0.98$ & 0.009 \\
Left ventricular ejection fraction & 0.86 & 4.22 & $0.75-0.98$ & 0.03 \\
Anxiety scores $\times$ PC & 1.4 & II.26 & $1.22-1.91$ & 0.006 \\
\hline
\end{tabular}

Note: Variables used in the model (age, gender, history of diabetes, history of HTN, history of smoking, history of previous AMI, beta blocker use, antianxiety use, aspirin use, left ventricular ejection fraction, anxiety scores, PC scores, and the interaction between anxiety and PC).

Abbreviations: AMI, acute myocardial infarction; HTN, hypertension; PC, perceived control.

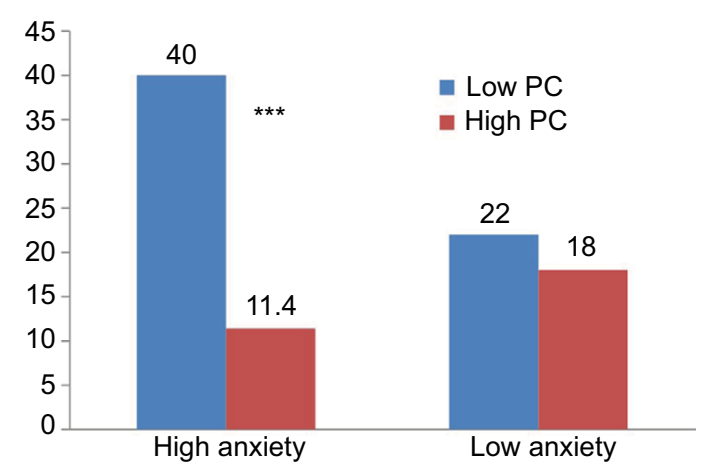

Figure 2 Comparison of percentage of patients who developed complications based on anxiety and PC.

Notes: Patients with high anxiety and low PC had the highest complication rate, indicating the moderating level of PC on the relationship between anxiety and complications. $* * * P<0.001$.

Abbreviation: PC, perceived control.

independent of sociodemographic and clinical variables, while PC has a protective effect against these complications.

This large sample study from a developing country gives further support to the hypothesis that PC is a moderating factor for the association between anxiety and in-hospital complications after AMI. Moreover, these results contribute to the body of knowledge that anxiety is a risk factor for in-hospital complications after AMI., ${ }^{2,3-8}$ However, some studies $^{5,16-18,31}$ did not show these relationships for numerous reasons, including the following: 1) failure to consider moderators of anxiety, 2) insufficient or small sample sizes, 3) timing of data collection for anxiety; 4) differences in the conceptual and operation definitions of anxiety (according to instruments); and 5) low rate of in-hospital complications among the patients.

For instance, McKinley et $\mathrm{al}^{5}$ used a sample of only 171 patients with acute coronary syndrome, $15 \%$ of whom developed in-hospital complications. This was expected 
in the study because the sample was mostly homogenous, comprising low-acuity angina patients. Approximately $82 \%$ of their sample was diagnosed with unstable angina, and only $18 \%$ was diagnosed with AMI. Moreover, only $5 \%$ of the sample who had heart failure presented with Killip class III and IV. The sample size of this study was not determined prior to data collection to make sure that it was sufficient. On the other hand, in the current study, and all other studies that showed a significant relation between anxiety and in-hospital complications among cohorts, all patients were diagnosed with AMI. ${ }^{6,8,10,13,15}$ Also, in Moser's study, ${ }^{10} 64 \%$ of the sample was diagnosed with STEMI; there was a higher percentage of patients with heart failure Killip class III and IV.

In this work and many previous studies, ${ }^{6,8,10,13,15}$ anxiety was measured in the hospital during the acute phase of illness. However, McKinley et $\mathrm{al}^{5}$ measured anxiety among outpatients or by telephone interviews while patients were at home. Patients with higher acuity levels are more prone to develop complications. The complication rate in the current study was $28 \%$ compared to differing prevalence in previous studies, including $15 \%,{ }^{5} 23.5 \%,{ }^{13} 27 \%,{ }^{10} 28 \%,{ }^{6}$ and $38.4 \%{ }^{2}$

Instruments measuring anxiety might include a broad anxiety symptom band. These symptoms go significantly beyond those of common physical illnesses, including subclinical CHD. In this study, anxiety was measured by HADS, which was originally proposed to detect anxiety in patients with physical illness. Therefore, it systematically excludes somatic symptoms which can imitate heart disease. ${ }^{32}$ Nevertheless, using this, some patients with mostly somatic symptoms of anxiety might go under detected. Consequently, this study is more likely to have underestimated (rather than overestimated) the association between anxiety and complications.

The results of this study demonstrate that $\mathrm{PC}$ had a protective effect against in-hospital complications after AMI. These results are consistent with a previous study ${ }^{10}$ checking the moderating effect of PC on anxiety and complications after AMI. It has been hypothesized that anxiety increased the rate of in-hospital complications through physiological and behavioral mechanisms. Behaviorally, anxiety might cause nonadherence to diet, smoking, lack of exercise, and motivation, ${ }^{10}$ but these factors are unlikely play a major role during an acute event. ${ }^{10}$ Physiologically, anxiety stimulates the sympathetic nervous system, resulting in a cascade of responses that increase the risk of complications (ie, decrease heart rate variability, increased platelet aggregation, and alteration in fibrillation threshold). ${ }^{2,3,6,10}$ These factors have been shown to play a major role in the development of complications early after AMI.,3,6,10
PC can alter this physiological response to anxiety by increasing coping responses under the conditions of threat. ${ }^{10,33}$ Pervious studies demonstrated that PC can alter the relationship between anxiety and in-hospital complication by its moderating effect. ${ }^{10,33}$ This means that when the PC is high, the association between the anxiety and complication is weak, and vice versa. High PC is associated with lower levels of anxiety, and therefore sympathetic nervous system stimulation is decreased, and the parasympathetic nervous activity increased. ${ }^{10,33}$ This change creates a cardioprotective effect and reduces in-hospital complications. The results of this study indicate that PC has a protective effect against complications, giving further support to its moderating effect.

PC can be enhanced by well-developed interventions, including teaching and psychotherapy. ${ }^{10,33}$ Such interventions can focus on restructuring the acute cardiac event (ie, AMI) from anxiety-producing stimulus to a condition that can be controlled by lifestyle changes and adherence to the recommended therapies. Randomized control trials checking the effect of PC in acute cardiac events are still warranted since such studies are still lacking in the clinical setting even in developed countries.

\section{Conclusion and clinical implications}

Anxiety is an independent predictor of in-hospital complications after STEMI. Perceived control had a moderator effect on this association, as evidenced by the highest rate of complication among the combination of high anxiety and low PC. Anxiety cannot be treated unless it is first recognized. Inclusion of simple tools to assess anxiety in hospital settings for patients experiencing AMI is highly recommended. It is possible to reduce the in-hospital complications after AMI generally, and especially for high-risk groups, by enhancing their PC levels. High levels of PC can decrease anxiety levels and therefore decrease the risk of complications.

\section{Disclosure}

The author reports no conflicts of interest in this work.

\section{References}

1. Benjamin EJ, Blaha MJ, Chiuve SE, et al. Heart disease and stroke statistics-2017 update: A report from the American Heart Association. Circulation. 2017;135(10):e146-e603.

2. AbuRuz ME, Masa'Deh R. Gender differences in anxiety and complications early after acute myocardial infarction. J Cardiovasc Nurs. 2017;32(6):538-543.

3. AbuRuz ME, Alaloul F, Al-Dweika G. Depressive symptoms are associated with in-hospital complications following acute myocardial infarction. Applied Nurs Res. 2018;39:65-70.

4. World Health Organization. Country Statistics and Global Health Estimates by WHO and UN Partner. Jordan: WHO Statistical Profile; 2015. Available from: http://www.who.int/countries/jor/en/. Accessed March 01, 2018. 
5. McKinley S, Fien M, Riegel B, et al. Complications after acute coronary syndrome are reduced by perceived control of cardiac illness. $J A d v$ Nurs. 2012;68(10):2320-2330.

6. Abu Ruz ME, Lennie TA, Moser DK. Effects of beta-blockers and anxiety on complication rates after acute myocardial infarction. Am J Crit Care. 2011;20(1):67-73; quiz 74.

7. Frasure-Smith N, Lesperance F, Talajic M. The impact of negative emotions on prognosis following myocardial infarction: is it more than depression? Health Psychol. 1995;14(5):388-398.

8. Moser DK, Dracup K. Is anxiety early after myocardial infarction associated with subsequent ischemic and arrhythmic events? Psychosom Med. 1996;58(5):395-401.

9. Benninghoven D, Kaduk A, Wiegand U, Specht T, Kunzendorf S, Jantschek G. Influence of anxiety on the course of heart disease after acute myocardial infarction - risk factor or protective function? Psychother Psychosom. 2006;75(1):56-61.

10. Moser DK, Riegel B, McKinley S, Doering LV, An K, Sheahan S. Impact of anxiety and perceived control on in-hospital complications after acute myocardial infarction. Psychosom Med. 2007;69(1):10-16.

11. Frasure-Smith N, Lesperance F. Depression and anxiety as predictors of 2-year cardiac events in patients with stable coronary artery disease. Arch Gen Psychiatry. 2008;65(1):62-71.

12. Roest AM, Martens EJ, Denollet J, de Jonge P. Prognostic association of anxiety post myocardial infarction with mortality and new cardiac events: a meta-analysis. Psychosom Med. 2010;72(6):563-569.

13. AbuRuz M, Demeh W. Anxiolytic medication use does not have a protective effect against complications after acute myocardial infraction. Life Sci J. 2013;10(4):1333-1337.

14. Abed MA, Kloub MI, Moser DK. Anxiety and adverse health outcomes among cardiac patients: a biobehavioral model. J Cardiovasc Nurs. 2014;29(4):354-363.

15. Abed MA, Frazier S, Hall LA, Moser DK. Anxiolytic medication use is not associated with anxiety level and does not reduce complications after acute myocardial infarction. J Clin Nurs. 2013;22(11-12):1559-1568.

16. Lane D, Carroll D, Ring C, Beevers DG, Lip GY. Effects of depression and anxiety on mortality and quality-of-life 4 months after myocardial infarction. J Psychosom Res. 2000;49(4):229-238.

17. Mayou RA, Gill D, Thompson DR, et al. Depression and anxiety as predictors of outcome after myocardial infarction. Psychosom Med. 2000;62(2):212-219.

18. Nakamura S, Kato K, Yoshida A, et al. Prognostic value of depression, anxiety, and anger in hospitalized cardiovascular disease patients for predicting adverse cardiac outcomes. Am J Cardiol. 2013;111(10):1432-1436.
19. Herrmann C, Brand-Driehorst S, Buss U, Ruger U. Effects of anxiety and depression on 5-year mortality in 5,057 patients referred for exercise testing. J Psychosom Res. 2000;48(4-5):455-462.

20. Moser DK, Riegel B, McKinley S, et al. The control attitudes scalerevised: psychometric evaluation in three groups of patients with cardiac illness. Nurs Res. 2009;58(1):42-51.

21. Heo S, Moser DK, Riegel B, Hall LA, Christman N. Testing a published model of health-related quality of life in heart failure. J Card Fail. 2005;11(5):372-379.

22. Moser DK, Dracup K. Psychosocial recovery from a cardiac event: the influence of perceived control. Heart Lung. 1995;24(4):273-280.

23. A-priori Sample Size Calculator for Multiple Regression. Available from: http://www.danielsoper.com/statcalc/calculator.aspx?id=1. Accessed October 13, 2017.

24. el-Rufaie OE, Absood GH. Retesting the validity of the Arabic version of the Hospital Anxiety and Depression (HAD) scale in primary health care. Soc Psychiatry Psychiatr Epidemiol. 1995;30(1):26-31.

25. el-Rufaie OE, Albar AA, Al-Dabal BK. Identifying anxiety and depressive disorders among primary care patients: a pilot study. Acta Psychiatr Scand. 1988;77(3):280-282.

26. el-Rufaie OE, Absood G. Validity study of the Hospital Anxiety and Depression Scale among a group of Saudi patients. Br J Psychiatry. 1987;151:687-688.

27. Bjelland I, Dahl AA, Haug TT, Neckelmann D. The validity of the Hospital Anxiety and Depression Scale. An updated literature review. J Psychosom Res. 2002;52(2):69-77.

28. Malasi TH, Mirza IA, el-Islam MF. Validation of the Hospital Anxiety and Depression Scale in Arab patients. Acta Psychiatr Scand. 1991;84(4):323-326.

29. World Health Organization. Process of Translation and Adaptation of Instruments. Geneva: World Health Organization. Available from: http://www.who.int/substance_abuse/research_tools/translation/en/. Accessed January 5, 2018.

30. Shieh G. Clarifying the role of mean centring in multicollinearity of interaction effects. Br J Math Stat Psychol. 2011;64(3):462-477.

31. Welin C, Lappas G, Wilhelmsen L. Independent importance of psychosocial factors for prognosis after myocardial infarction. J Intern Med. 2000;247(6):629-639.

32. Zigmond AS, Snaith RP. The hospital anxiety and depression scale. Acta Psychiatr Scand. 1983;67(6):361-370.

33. Moser DK, Dracup K. Impact of cardiopulmonary resuscitation training on perceived control in spouses of recovering cardiac patients. Res Nurs Health. 2000;23(4):270-278.
Journal of Multidisciplinary Healthcare

\section{Publish your work in this journal}

The Journal of Multidisciplinary Healthcare is an international, peerreviewed open-access journal that aims to represent and publish research in healthcare areas delivered by practitioners of different disciplines. This includes studies and reviews conducted by multidisciplinary teams as well as research which evaluates the results or conduct of such teams or health

\section{Dovepress}

care processes in general. The journal covers a very wide range of areas and welcomes submissions from practitioners at all levels, from all over the world. The manuscript management system is completely online and includes a very quick and fair peer-review system. Visit http://www.dovepress.com/ testimonials.php to read real quotes from published authors. 